\title{
Using hybrid transcription factors to study gene function in rice
}

\author{
ZHAO Tao ${ }^{1 \dagger}$, LIU Jun $^{1 \dagger}$, LI Hong-Yu ${ }^{1 \dagger}$, LIN Jian-Zhong ${ }^{2 \dagger}$, BIAN Ming-Di ${ }^{3 \dagger}$, ZHANG \\ Chun-Yu ${ }^{1}$, ZHANG Yong-Xing ${ }^{1}$, PENG Yu-Chong ${ }^{2}$, LIU Bin ${ }^{1 *} \&$ LIN ChenTao ${ }^{4}$ \\ ${ }^{1}$ Institute of Crop Sciences, Chinese Academy of Agricultural Sciences, Beijing 100081, China; \\ ${ }^{2}$ College of Biology, Hunan Province Key Laboratory of Plant Functional Genomics and Developmental Regulation, Hunan University, \\ Changsha 410082, China; \\ ${ }^{3}$ Laboratory of Soil and Plant Molecular Genetics, College of Plant Science, Jilin University, Changchun 130062, China; \\ ${ }^{4}$ Department of Molecular, Cell \& Developmental Biology, University of California, Los Angeles, CA90095, USA
}

Received September 14, 2015; accepted September 18, 2015; published online September 24, 2015

Citation: Zhao T, Liu J, Li HY, Lin JZ, Bian MD, Zhang CY, Zhang YX, Peng YC, Liu B, Lin CT. Using hybrid transcription factors to study gene function in rice. Sci China Life Sci, 2015, 58: 1160-1162, doi: 10.1007/s11427-015-4937-x

Dear Editors,

Transcription factors (TF) play important roles in plant growth and development, which determine crop productivity, quality, and stress tolerance. However, the basic mechanism governing transcription regulation by $\mathrm{TF}$ remains largely unclear in plants. It is well known that TF proteins contain at least two functional domains, the DNA-binding domain and the transcription regulatory domain. The VP16 transcription activation domain of human Herpes simplex virus and the EAR transcription suppression domain of many plant TF proteins are two of the best-known transcription regulatory domains $[1,2]$. It has been reported that both VP16 and EAR domains exert their respective transcription activation/repression functions in heterologous protein and/or cellular environment [2-4], supporting a hypothesis that the transcription regulatory domains function independent of other functional domains of TF proteins. However, this hypothesis has not been examined systematically. We reasoned that a phenotypic analysis of transgenic expression of all or most TF genes from a plant species in the form of fusion proteins, which are composed of the coding sequences of TFs fused to the VP16 or EAR domain, would allow a systematic test of this hypothesis, and we reported

$\dagger$ Contributed equally to this work

*Corresponding author (email: liubin05@caas.cn) here the overall result of such an experiment.

We cloned cDNAs for 1,685 out of the 3,148 predicted transcription factor genes from rice (Oryza sativa subsp. Japonica) (Figure 1A, Table S1 in Supporting Information), and prepared 2,980 HTF Ti plasmid constructs that express the EAR4 (tetrameric repeats of EAR)-TF fusion proteins or the VP64 (tetrameric repeats of VP16)-TF fusion proteins under the control of the maize ubiquitin promoter [5] (Figure 1B, Table S2 in Supporting Information). We obtained 57,751 independent transgenic lines in the Japonica rice accession Kitaake that express a total of 2,470 HTFs constructs. On average, 23 independent transgenic lines were generated per construct (Figure 1C, Table S3 in Supporting Information). Approximately 13\% (7,719/57,751 events) of transgenic lines showed phenotypic alterations in comparison to the wild-type parents, including changes in morphology, heading date, or grain yield (Figure S1 in Supporting Information). Expression of 957 HTF fusion proteins, including 343 EAR4 HTF and 614 VP64 HTF proteins, caused the phenotypic alteration in at least three independent transgenic lines, and they were investigated in more details (Figure 1D). 132 pairs of these 957 HTFs exhibited phenotypic alterations in both the EAR4 and VP64 configurations, of which 86, 30 and 16 pairs of EAR4 and VP64 HTFs resulted in similar, different, or opposite phenotypes, respectively (Figure 1D, Pie diagram). It is surprising that only $12 \%(16 / 132)$ of EAR4- and VP64-fusion 


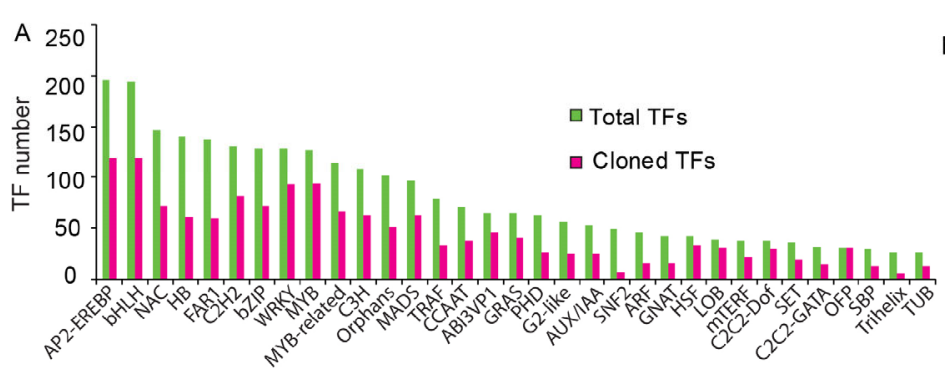

C
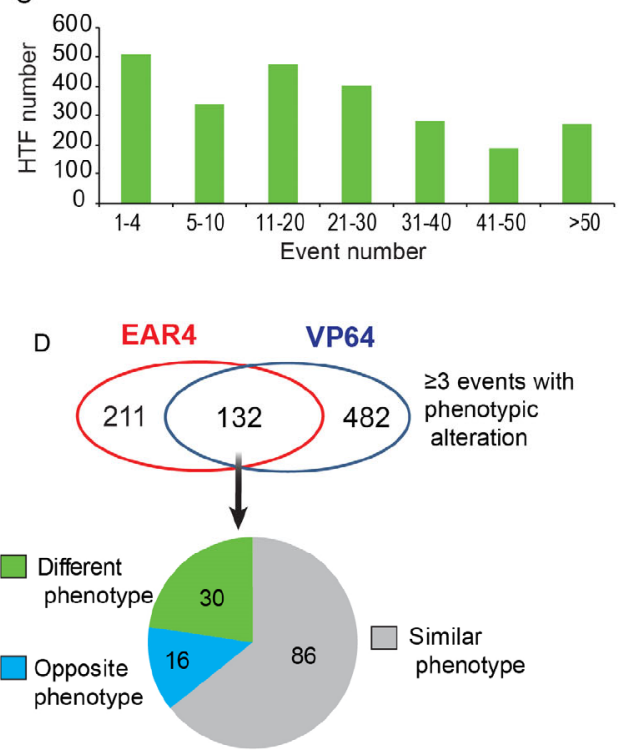

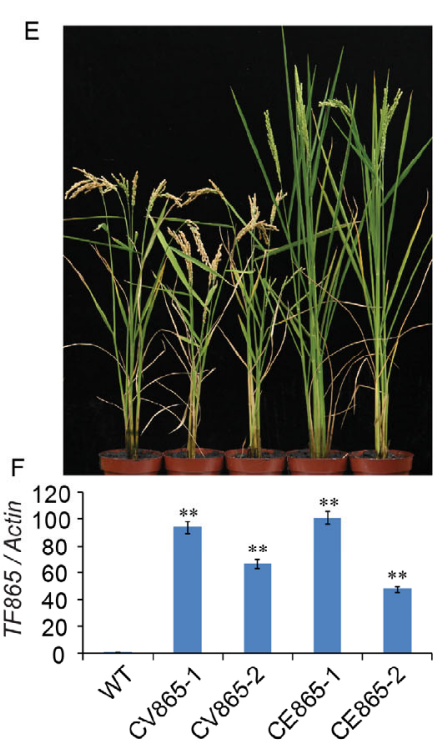

B
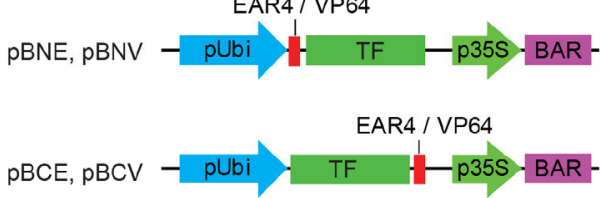

EAR4 / VP64

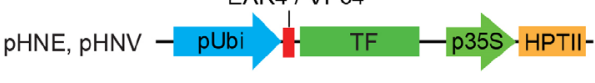

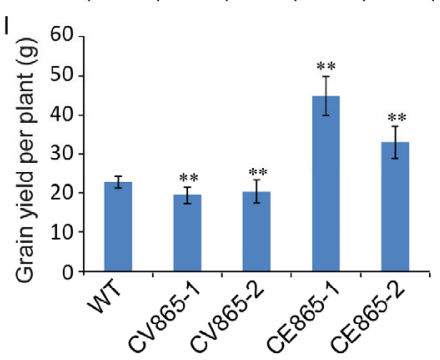

Figure 1 Using hybrid transcription factors to identify gene functions. A, The distribution of cloned TFs in different gene families. B, Schematic presentations of the HTF constructs. The detailed vector maps and sequences are documented in Supporting Information. C, Transgenic event number distribution of the HTF transgenes. D, An overview of constructs with at least 3 transgenic events showing similar phenotypic alteration. E, Representative image of $C V 865$ and CE865 transgenic plants at adult stage. F, qRT-PCR analysis showing the overexpression of $C V 865$ and $C E 865$ mRNA in each transgenic line. G, Immunoblot showing the level of CV865 protein in the indicated lines. The CV865 protein was detected with anti-VP16 antibody. The ponceau staining band of Rubisco large subunit was used as loading control. H and I, Statistical analysis of plant height (H) and grain yield per plant (I) of indicated genotypes, means $\pm \mathrm{SD}(* *, P<0.001, n>20)$ are shown.

proteins caused opposite phenotypes, suggesting that the activity of a transcription regulatory domain is more dependent on other functional domains or the structural context than what the previous reports may have suggested. Exactly how a transcription regulatory domain is dependent on other functional domains or the structural context of the HTF proteins remains unclear, but the speculative interpretations would include distinct structural impacts of the VP64 or EAR4 modules, different functional effects of fusion protein configuration to the activity of individual module, distinct modes of action of the two modules, and so on. For example, VP16 is known to interact with not only specific host transcription factors, such as Oct-1 and HCF-1[6], but also components of general transcription machinery, such as TBP, TFIIB, and TFIIH [7,8], whereas EAR is only known to interact with specific transcription regulators, such as TPL, to recruit histone deacetylases [9]. It is conceivable that VP64-fusion proteins are more likely to interact with the general transcription machinery to activate transcription in a heterologous protein and cellular environment, whereas the EAR4-fusion proteins may be less likely to encounter and interact with the specific transcription regulators to suppress transcription in the heterologous protein environment.

On the other hand, those 16 HTFs that conferred opposite phenotypes in the VP64 or EAR4 configuration provide a relatively simpler explanation for the mode of action of the respective rice TF genes (Table S4 in Supporting Information). For example, a MYB-like transcription factor (encoded by LOC_OsO6g45890), referred to as TF865 in this study, showed opposite growth phenotype in the EAR4 or VP64 configuration. Transgenic lines expressing the VP64-fusion proteins (CV865) exhibited dwarf phenotype, whereas those expressing the EAR4-fusion proteins (CE865) grew significantly taller than the wild-type control (Figure 1E and 1H). Overexpression of both CV865 and CE865 transgenes were confirmed by the quantitative reverse transcription PCR (qRT-PCR) or the immunoblot 
analyses (Figure $1 \mathrm{~F}$ and $1 \mathrm{G}$ ). In comparison to that of the wild-type control, the grain yield per plant of transgenic lines expressing the EAR4-fusion protein (CE865) or the VP64-fusion protein (CV865) increased $(50 \%-100 \%)$ or decreased $(10 \%-20 \%)$, respectively (Figure 1I). These results are consistent with the hypothesis that this MYB-like $\mathrm{TF}$ gene may regulate rice growth and development or metabolism to affect grain yield. Further study of other HTF that caused various phenotypic variations will likely reveal the functional roles and modes of action for other rice TF genes. Transcription factors regulate almost all aspects of biological processes by dynamically modulating target gene transcription. A large percentage of genes, ranged from $1 \%-6 \%$ in different Eukaryotic species, encode TFs [10]. Only a relatively small fraction of eukaryotic TFs' DNA sequence preferences are experimentally determined, but their biological functions are much less determined. The functional redundancy of many plant TF genes imposes a major technical difficulty in the study of physiological roles of many TF genes. The HTF transgenic resource reported here provides a valuable tool complement to the loss-of-function resources reported previously for the functional study of rice genes.

This work is supported by the MOA Transgenic Research Grant (2010ZX08010-002 to ICS-CAAS), Agricultural Science and Technology Innovation Program of CAAS, and National Natural Science Foundation of China (31422041, 31371649, 31301298 and 31170172).
1 Sadowski I, Ma J, Triezenberg S, Ptashne M. GAL4-VP16 is an unusually potent transcriptional activator. Nature, 1988, 335: 563-564

2 Kagale S, Rozwadowski K. EAR motif-mediated transcriptional repression in plants: an underlying mechanism for epigenetic regulation of gene expression. Epigenetics, 2011, 6: 141-146

3 Ohta M, Matsui K, Hiratsu K, Shinshi H, Ohme-Takagi M. Repression domains of class II ERF transcriptional repressors share an essential motif for active repression. Plant Cell, 2001, 13: 1959-1968

4 Herve C, Dabos P, Bardet C, Jauneau A, Auriac MC, Ramboer A, Lacout F, Tremousaygue D. In vivo interference with AtTCP20 function induces severe plant growth alterations and deregulates the expression of many genes important for development. Plant Physiol, 2009, 149: 1462-1477

5 Cornejo MJ, Luth D, Blankenship KM, Anderson OD, Blechl AE. Activity of a maize ubiquitin promoter in transgenic rice. Plant Mol Biol, 1993, 23: 567-581

6 Wysocka J, Herr W. The herpes simplex virus VP16-induced complex: the makings of a regulatory switch. Trends Biochem Sci, 2003, 28: 294-304

7 Xiao H, Pearson A, Coulombe B, Truant R, Zhang S, Regier JL, Triezenberg SJ, Reinberg D, Flores O, Ingles CJ, et al. Binding of basal transcription factor TFIIH to the acidic activation domains of VP16 and p53. Mol Cell Biol, 1994, 14: 7013-7024

8 Hall DB, Struhl K. The VP16 activation domain interacts with multiple transcriptional components as determined by protein-protein cross-linking in vivo. J Biol Chem, 2002, 277: 46043-46050

9 Wang L, Kim J, Somers DE. Transcriptional corepressor TOPLESS complexes with pseudoresponse regulator proteins and histone deacetylases to regulate circadian transcription. Proc Natl Acad Sci USA, 2013, 110: 761-766

10 Jin J, Zhang H, Kong L, Gao G, Luo J. PlantTFDB 3.0: a portal for the functional and evolutionary study of plant transcription factors. Nucleic Acids Res, 2014, 42: D1182-1187

Open Access This article is distributed under the terms of the Creative Commons Attribution License which permits any use, distribution, and reproduction in any medium, provided the original author(s) and source are credited.

\section{Supporting Information}

Figure S1 The number of HTFs in EAR4 or VP64 configuration showing indicated phenotypic alterations.

Table S1 The list of 1685 transcription factor genes cloned into pDONR vector.

Table S2 The list of 2980 HTF constructs.

Table S3 The numbers of independent transgenic events of each HTF construct.

Table S4 The list of 16 pairs of EAR4- and VP64-fusion proteins causing opposite phenotypes.

The supporting information is available online at life.scichina.com and link.springer.com. The supporting materials are published as submitted, without typesetting or editing. The responsibility for scientific accuracy and content remains entirely with the authors. 